\title{
On Acoustic Radiation by Rotating Dipole Sources in Frequency Domain
}

\author{
Zhihong Liu \\ Center of Energy and Environment, QingDao Technological University \\ 11 FuShun Road, QingDao 266033, China \\ Tel: 86-136-1648-9849Ｅ-mail: lzhqingdao@163.com
}

Chuijie Yi

Center of Energy and Environment, QingDao Technological University

E-mail: Chuijieyi@vip.163.com

Received: May 18, 2011

Accepted: July 23, 2011

doi:10.5539/mas.v5n5p80

\begin{abstract}
In order to deal with the acoustic radiation from rotating sources, this paper describes a frequency-domain numerical method for predicting sound radiation. The method is based on the analytical Green's function of rotating monopole and dipole source in free space. Sound radiation model is established and characteristics of sound field are discussed by numerical simulation and the relationship between radiated sound frequencies and acoustic nature frequency of source, angular frequency and its harmonics can be revealed .The radiated sound field has a strong directivity, fundamental frequency transmitting in the rotary shaft direction and harmonics spreading along radial direction and frequency shift phenomena appearing clearly in higher rotating speed of source. The method has a theoretical significance for exploring the low-noise rotating machinery.
\end{abstract}

Keywords: Acoustic radiation, Rotating dipole sources, Analytical green's function

\section{Introduction}

Due to the relative motion between medium and sound source, the sound radiation of moving source has become the difficulty in acoustic domain. More attention to the complexity of sound generation of moving sound sources has been paid by some authors. P.M.Morse and K.U.Zngard have been discussed sound pressure of linear motion point source using the coordinate transform. This research is only available for simple moving sources and for the complex motion source the coordinate transform is difficult or impossible. M.V.Lowson derived firstly acoustic equations from rotating point force .The equations is applied to predict the sound radiation of rotor and the prediction pressure is exact, but the equation is only used to rotor in free field and in fact the rotor is often in case or in box, the effect from the boundaries is neglect. This will give more inaccurate for the prediction pressure. In order to overcome the disadvantage, K.D.Hand D.J.Leeh as given sound field equations of rotating point force with cylindrical boundary using direct boundary element method, Helmholtz equation and its normal derivative and W.H.Jeon and D.J.Lee extended this research, discussed centrifugal fan features of the sound source and forecasting method and Han-Lim Choid and D. J. Lee began to study rotating acoustic source radiation in open thin wall pipe. These methods have established the foundation for sound radiation of moving source, but there are some problems needed to overcome further. The assumption of point source and plane wave simplify the fact but the more inaccurate for the prediction will bring. Moreover, the sound pressure equations are often given in time domain. The time model is difficult to calculate and obtain the numerical solution. In order to deal with the problems above, in the present paper the analytical expressions of the pressure of rotating dipole in frequency domain based on numerical Green's function is derived in section2. Characteristics of Sound field are given by numerical simulation in section3. The results are presented in section4.The analytical method provided important theoretical value for the research of sound radiation and noise control of moving source.

\section{Analytical formulae of rotating dipole sources}

In this section, the analytical Green's function and sound pressure model of rotating dipole sources is derived in the frequency domain. Rotating dipole source's analytical Green's function and sound field expressions in frequency domain based on the rotating point source Green's function is derived. Two dipole form, the horizontal 
(along the $\varphi$ orientation) and vertical directions (along the $\mathrm{z}$ axis), are discussed. Two point sources of opposite phase, as shown in Figure.1 and Figure. 2.

We can get multipole field by the partial derivatives of free-field rotating point source's Green's function that are taken with respect to the source coordinates. By taking the Green formula as a common starting point.

\subsection{Free-field analytical Green's function}

The Green's function $G(x, t \mid y, \tau)$ is defined as the response of the flow to a impulsive point source represented by delta functions of space and time:

$$
\frac{1}{c_{0}^{2}} \frac{\partial^{2} G}{\partial t^{2}}-\nabla^{2} G=\delta(\vec{x}-\vec{y}) \delta(t-\tau)
$$

Where $\delta(x-y)=\delta\left(x_{1}-y_{1}\right) \delta\left(x_{2}-y_{2}\right) \delta\left(x_{3}-y_{3}\right)$, when $t<\tau$, then $G=0$.

$$
G(\vec{x}, \vec{y}, t, \tau)=\frac{1}{4 \pi|\vec{x}-\vec{y}|} \delta\left(t-\tau-\frac{|\vec{x}-\vec{y}|}{c_{0}}\right)=\frac{1}{4 \pi r_{s}} \delta\left(t-\tau-\frac{r_{s}}{c_{0}}\right)
$$

Where $(\vec{x}, t)$ are for the observer's position $\vec{x}$ at time $t,(\vec{y}, \tau)$ are for the source's location and time $r_{s}=|\vec{x}-\vec{y}(\tau)|$, $c_{0}$ are for acoustic velocity. $\hat{G}$ is the Fourier transformed Green's function defined by

$$
\hat{G}=\frac{1}{2 \pi} \int_{-\infty}^{\infty} G(x, t / y, \tau) e^{-i \omega t} d t
$$

Taking the Fourier transform on both sides of the formula (2) can get Green's function in frequency-domain

$$
\hat{G}(\vec{x}, \vec{y}, \omega, \tau)=\frac{1}{8 \pi^{2} r_{s}} e^{-i \omega t} e^{i \kappa r_{s}}
$$

Where $\omega$ is nature frequency of source, $\kappa=\omega / c_{0}$ is wave number.

Expanding formula (3) by Legendre and Spherical harmonic functions in the spherical coordinates:

$$
\frac{e^{i k_{s}}}{4 \pi r_{s}}=i \kappa \sum_{n=0}^{\infty} b_{n} \sum_{m=-n}^{n} Y_{n}^{m *}(\theta, \varphi) Y_{n}^{m}\left(\theta_{0}, \varphi_{0}\right)=\frac{i \kappa}{4 \pi} \sum_{n=0}^{\infty}(2 n+1) b_{n} P_{n}(\cos \beta)
$$

By the Euler's formula and addition theorem

$$
P_{n}(\cos \beta)=\sum_{m=0}^{n} \varepsilon_{m} a_{m n}\left[e^{i m\left(\varphi-\varphi_{0}\right)}+e^{-i m\left(\varphi-\varphi_{0}\right)}\right]=\sum_{m=0}^{n} \varepsilon_{m} a_{m n}\left[e^{i m \varphi_{0}} e^{-i m \Omega \tau}+e^{-i m \varphi_{0}} e^{i m \Omega \tau}\right]
$$

Where $b_{n}=\left\{\begin{array}{ll}j_{n}\left(\kappa r_{0}\right) h_{n}(\kappa r) & r>r_{0} \\ j_{n}(\kappa r) h_{n}\left(\kappa r_{0}\right) & r<r_{0}\end{array}, \varepsilon_{m}=\left\{\begin{array}{cc}1 / 2 & m=0 \\ 1 & m=1 \cdots n\end{array}, a_{m n}=\frac{(n-m) !}{(n+m) !} P_{n}^{m}(\cos \theta) P_{n}^{m}\left(\cos \theta_{0}\right)\right.\right.$

$\beta$ is the angle between the vector $r_{0}$ and $r$.

Taking formula (4) into (3), Green's function of rotating point source in free space is given by

$$
\hat{G}(\vec{x}, \vec{y}, \omega, \tau)=\frac{i \kappa}{8 \pi^{2}} \sum_{n=0}^{\infty} \sum_{m=0}^{n} \varepsilon_{m}(2 n+1) b_{n} a_{m n}\left(e^{-i m \varphi_{0}} e^{i(\omega+m \Omega) \tau}+e^{i m \varphi_{0}} e^{-i(\omega-m \Omega) \tau}\right)
$$

Vertical Green's function of rotating dipole source is defined the derivatives of $\hat{G}$ that is taken with respect to the source coordinates of the direction of $Z(\theta=0)$, which is shown as below:

$$
\begin{gathered}
\hat{G}_{z}=-\partial \hat{G} / \partial z=-(\partial \hat{G} / \partial r) \cos \theta \\
\hat{G}_{Z}\left(r / r_{0}\right)=\sum_{n=0}^{\infty} \sum_{m=0}^{n} \varepsilon_{m}\left(-i \kappa^{2}\right)\left(\frac{(2 n+1)}{8 \pi^{2}}\right) \cos \theta b_{n}^{\prime} a_{m n}\left(e^{-i m \varphi_{0}} e^{-i(\omega+m \Omega) \tau}+e^{i m \varphi_{0}} e^{-i(\omega-m \Omega) \tau}\right)
\end{gathered}
$$

Where $b_{n}^{\prime}=\left\{\begin{array}{ll}j_{n}\left(\kappa r_{0}\right) h_{n}^{\prime}(\kappa r) & r>r_{0} \\ j_{n}^{\prime}(\kappa r) h_{n}\left(\kappa r_{0}\right) & r<r_{0}\end{array}\right.$. 
Horizontal Green's function of dipole source is the derivatives of $\hat{G}$ along the $\varphi$ orientation

$$
\begin{gathered}
\hat{G}_{\varphi}=-\frac{1}{r_{0}} \frac{\partial \hat{G}}{\partial \varphi} \\
\hat{G}_{\varphi}\left(r / r_{0}\right)=\sum_{n=0}^{\infty}\left(-i \kappa / r_{0}\right)\left[\frac{(2 n+1)}{8 \pi^{2}}\right] b_{n} * \\
\left\{P_{n}(0) P_{n}(\theta)-\sum_{m=1}^{n} \frac{(n-m) !}{(n+m) !} P_{n}^{m}(0) P_{n}^{m}(\theta) i m\left[e^{-i m \varphi_{0}} e^{-i(\omega+m \Omega) \tau}-e^{i m \varphi_{0}} e^{-i(\omega-m \Omega) \tau}\right]\right\}
\end{gathered}
$$

\subsection{Sound pressure model and Directivity}

\subsubsection{Sound pressure of rotating dipole source}

On the basis of the assumption, the acoustic field is for a given time-harmonic source distribution $Q_{s}(\tau)=Q(\tau) e^{-i \omega \tau}$.

Sound pressure is given by

$$
p(\vec{r}, t)=\int_{-\infty}^{\infty} Q_{s}(\tau) G d \tau=\int_{-\infty}^{\infty} Q_{s}(\tau) \frac{1}{4 \pi R} \delta\left(t-\tau-\frac{R}{c}\right) d \tau
$$

Taking the Fourier transform on both sides of the formula (9) can get sound pressure in frequency-domain

$$
p(\vec{r}, \omega)=\frac{1}{2 \pi} \int_{-\infty}^{\infty} \int_{-\infty}^{\infty} Q_{s}(\tau) G e^{-i \omega t} d \tau d t=\frac{1}{2 \pi} \int_{-\infty}^{\infty} Q(\tau) e^{-i \omega \tau} \hat{G} d \tau
$$

Where $Q(\tau)$ is point source strength, Assumption the $A_{0}(\omega)$ is the Fourier transform of $Q(\tau)$, then

$$
A_{0}(\omega)=\frac{1}{2 \pi} \int_{-\infty}^{\infty} Q(\tau) e^{-i \omega \tau} d \tau
$$

For a given time-harmonic source, formula (11) is rewrite as

$$
A_{0}(\omega)=Q \delta\left(\omega-\omega_{t}\right)
$$

Where $\omega_{t}$ is the source natural frequency.

The sound pressure of rotating dipole sources are presented

$$
\begin{gathered}
p_{z}=D_{s} \sum_{n=0}^{\infty} \sum_{m=0}^{n} \varepsilon_{m}\left(-i \kappa^{2}\right)\left(\frac{(2 n+1)}{8 \pi^{2}}\right) \cos \theta_{0} B_{n}^{\prime} \frac{(n-m) !}{(n+m) !} * P_{n}^{m}(0) P_{n}^{m}\left(\cos \theta_{0}\right) *\left[\left(e^{i m\left(-\varphi_{0}\right)} \delta\left(\omega+m \Omega-\omega_{t}\right)\right)\right. \\
\left.+\left(e^{-i m\left(-\varphi_{0}\right)} \delta\left(\omega-m \Omega-\omega_{t}\right)\right)\right] \\
p_{\varphi}=D_{s} \sum_{n=0}^{\infty} \frac{-i \kappa(2 n+1) B_{n}}{8 \pi^{2} r_{0}}\left\{\begin{array}{c}
P_{n}(0) P_{n}\left(\cos \theta_{0}\right)-\sum_{m=1}^{n} i m \frac{(n-m) !}{(n+m) !} P_{n}^{m}(0) P_{n}^{m}\left(\cos \theta_{0}\right) * \\
\left.\left(e^{i m\left(-\varphi_{0}\right)} \delta\left(\omega+m \Omega-\omega_{t}\right)+e^{-i m\left(-\varphi_{0}\right)} \delta\left(\omega-m \Omega-\omega_{t}\right)\right)\right\}
\end{array}\right.
\end{gathered}
$$

We defined the dipole strength $D_{s}=Q_{s} d$, and assume that $d$ is vanishingly small.

2.2.2 Directivity of rotating dipole source

The far-field directivity pattern defined by removing the $e^{-i \kappa r} / r$ factor so that the directivity pattern $D(\theta, \varphi)$ is defined by

far-field directivity of rotating dipole source

$$
\lim _{x \rightarrow \infty}[p(r, \theta, \varphi)]=\left(e^{-i \kappa r} / r\right) D(\theta, \varphi)
$$

$$
D_{z}(\theta, \varphi)=r e^{-i \kappa r} G_{z}, \quad D_{\varphi}(\theta, \varphi)=r e^{-i \kappa r} G_{\varphi}
$$




\section{Numerical Analysis of Sound field}

Acoustic field characteristics are given in the condition of time-harmonic source. Simulation parameters: Azimuth of observation point $\varphi_{0}=-\pi / 4$, source nature frequencies $\omega_{t}$ are respectively $2300 \mathrm{~Hz}$ and $6800 \mathrm{~Hz}$,rotating angular frequencies $\Omega$ are $112 \mathrm{rad} / \mathrm{s}$ and $560 \mathrm{rad} / \mathrm{s}$, acoustic field frequency is shown $\omega=\omega_{t}+k \Omega, k$ is harmonic orders for arbitrary integer that sets $k=-5, \cdots, 5$.For symbol concision in figures using $\omega_{0}$ substitute for $\Omega$ and $Y$ substitute for $\theta_{0}$ that are evaluated with $0, \pi / 18, \pi / 6, \pi / 3, \pi / 2$ respectively. For far-field $r=2 m$ and $r_{0}=0.3 m$.Simulation results are demonstrated in Figure. 3 and Figure.4.

(1) In Figure.3, with the increasing of nature frequencies, the far-field sound pressure amplitude of vertical dipole increases, the scope of harmonic distribution expand and fundamental frequency component reduce as the observation angle augmentation. Harmonic distribution is the most abundant in the observation angle of $\pi / 2$.(2) There is only fundamental frequency in the rotating axis direction .(3) With the increasing of angular frequencies, the distribution of harmonics and sound pressure amplitude increase.

(4)In Figure.4 sound pressure amplitude is impacted greatly by the nature frequencies. the scope of harmonic distribution expands with the increasing of view angle.(5) Fundamental frequency is evidence in the small observation angle as 0 and $\pi / 18$.there is no harmonic orders in the observation angle of $\pi / 2$. (6) The nature and angular frequencies changing have great effect on the harmonic distribution. Rotating dipole sources have an intensive space directivity that are shown in Figure.5 and Figure.6. With the increasing of harmonic orders, the directivity of vertical dipole becomes intensive. Especially, sound power focuses on the range from $\pi / 6$ to $\pi / 3$ in Figure.5. In Figure.6 with the increasing of harmonic orders, the directivity of horizontal dipole becomes intensive. Especially, sound power focuses on the range from $\pi / 3$ to $\pi / 2$. The distribution from horizontal and vertical rotating dipole source at different observation angle is shown by Figure7.The total sound pressure is mainly from horizontal rotating dipole source.

\section{Conclusions}

A frequency-domain analytical method has been developed for predictiong sound field of rotating diople source.The method is based on analytical Green's function in freqency-domain.Sound pressure model and the characteristics of sound field of moving source are discussed. By numerical simulations we can get that the sound field frequecy involves nature frequency, angular frequency and its harmonics ,the distribution from horizontal and vertical rotating dipole source, and The intensive directivity. The method and results have important theoretical significance on the moving source sound field characteristic analysis and exploring the low-noise design of rotary machine.

\section{References}

Han-Lim Choi,D. J. Lee. (2006). Development of the numerical method for calculating sound radiation from a rotating dipole source in an opened thin duct. Journal of Sound and Vibration,295:739-752. doi:10.1016/j.jsv.2006.01.017, http://dx.doi.org/10.1016/j.jsv.2006.01.017

K.D. Ih, D.J. Lee. (1997). Development of the direct boundary element method for thin bodies with general boundary conditions. Journal of Sound and Vibration, 202:361-373.

LiuQiuhong,QiDatong. (2004). A unmberical method and a aeroacostical model of the centrifugal fan. Journal of XiAn Jiao'Tong University, 138(3):313-316.

Lowson M V. (1965). The sound field for singularities in motion. Proceeding of the Royal Society in London, Series A,286:559-572.

P. M.Morse ,K. U. Ingard. (1968). Theoretical Acoustics, McGraw-Hil. New York.

W. H. Jeon, D. J. Lee. (2000). An analysis of generation and radiation of sound for a centrifugal fan. in: Seventh ICSV,121(3):1235-1242.

W.H. Jeon, D.J. Lee. (2000). An analysis of the flow and aerodynamic acoustic sources of a centrifugal impeller. Journal of Sound and Vibration, 222:505-511. doi:10.1006/jsvi.1998.1983, http://dx.doi.org/10.1006/jsvi.1998.1983

W.H. Jeon, D.J. Lee. (2003). A numerical study on the flow and sound fields of centrifugal impeller located near a wedge. Journal of Sound and Vibration, 266:785-804.

WuJiuhui,ChenHualing. (2000). Acoustic Solution of Rotating Point Source in Frequency Domain. Journal of XiAn Jiao'Tong University, 34(1):71-75. 
Zhihongliu, Chuijieyi,et al. (2009). Research for Characteristics of Rotating Dipole Acoustic Source in Spatial Acoustic Field. Modern Applied Science,3(6):31-39.

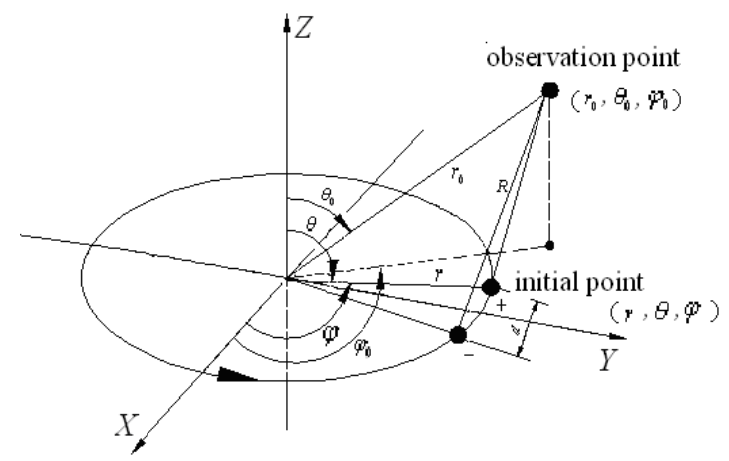

Figure 1. Horizontal rotating dipole source

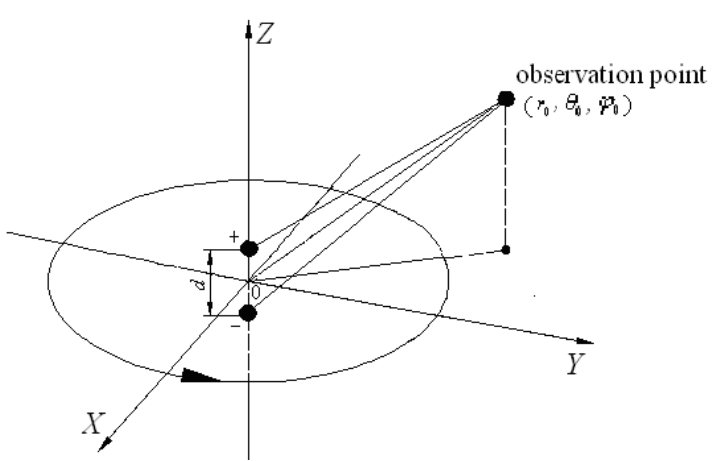

Figure 2. Vertical rotating dipole source

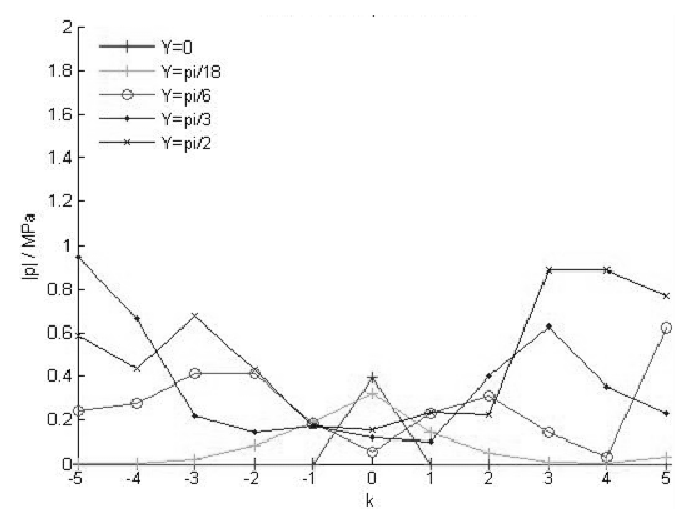

(a) $\omega_{t}=6800 \mathrm{~Hz}, \omega_{0}=560 \mathrm{rad} / \mathrm{s}$

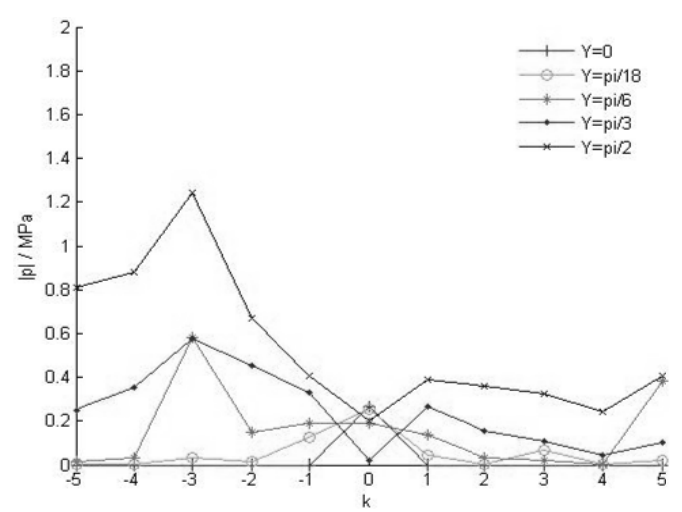

(c) $\omega_{t}=2300 \mathrm{~Hz}, \omega_{0}=560 \mathrm{rad} / \mathrm{s}$

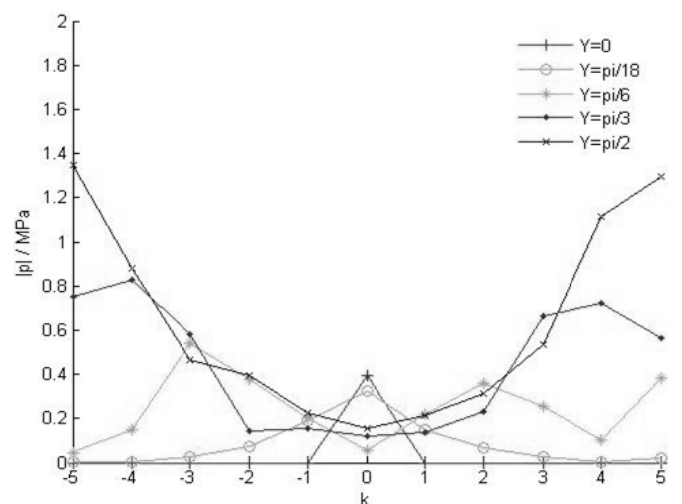

(b) $\omega_{t}=6800 \mathrm{~Hz}, \omega_{0}=112 \mathrm{rad} / \mathrm{s}$

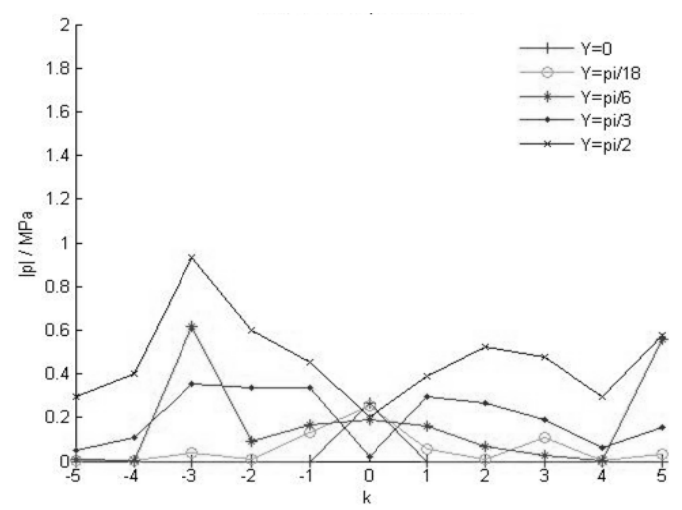

(d) $\omega_{t}=2300 \mathrm{~Hz}, \omega_{0}=112 \mathrm{rad} / \mathrm{s}$

Figure 3. Sound pressure amplitude distribution of vertical dipole with observation angle at different nature and angular frequencies 


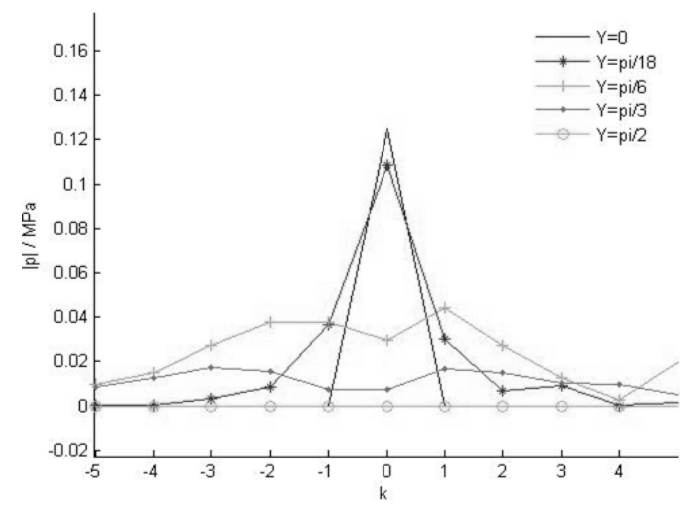

(a) $\omega_{t}=6800 \mathrm{~Hz}, \omega_{0}=560 \mathrm{rad} / \mathrm{s}$

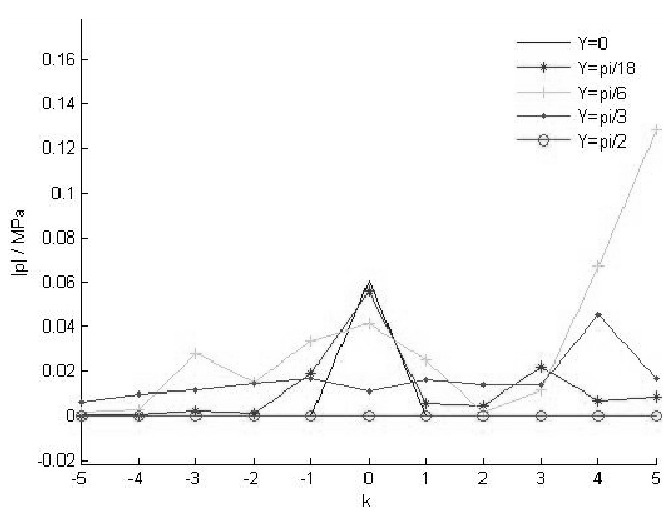

(c) $\omega_{t}=2300 \mathrm{~Hz}, \omega_{0}=560 \mathrm{rad} / \mathrm{s}$

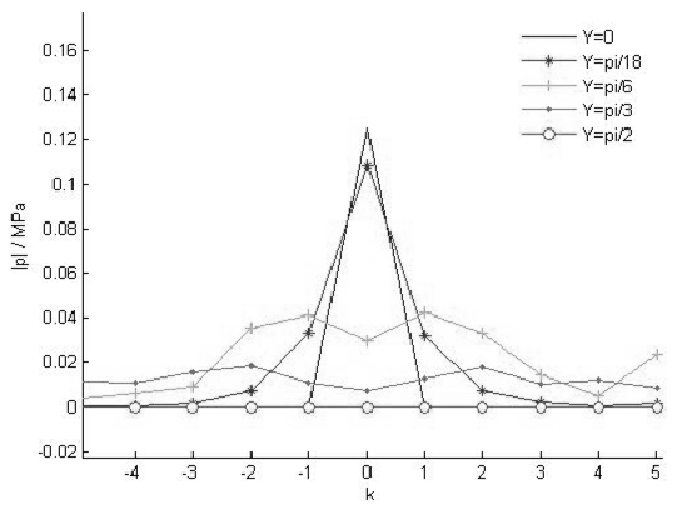

(b) $\omega_{t}=6800 \mathrm{~Hz}, \omega_{0}=112 \mathrm{rad} / \mathrm{s}$

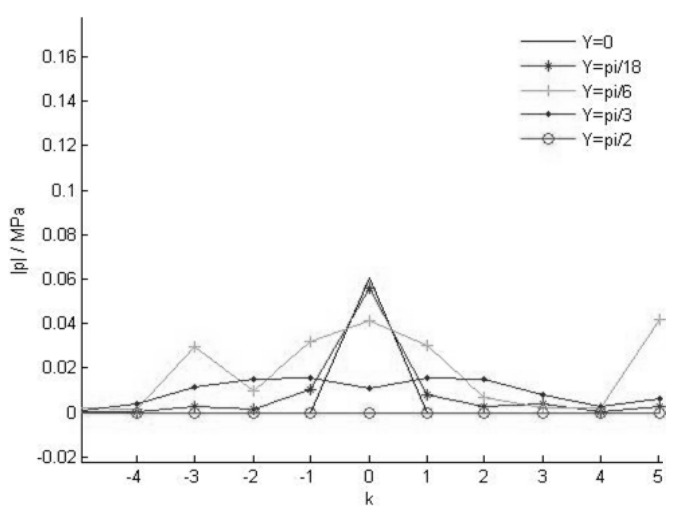

(d) $\omega_{t}=2300 \mathrm{~Hz}, \omega_{0}=112 \mathrm{rad} / \mathrm{s}$

Figure 4. Sound pressure amplitude distribution of horizontal dipole with observation angle at different nature and angular frequencies

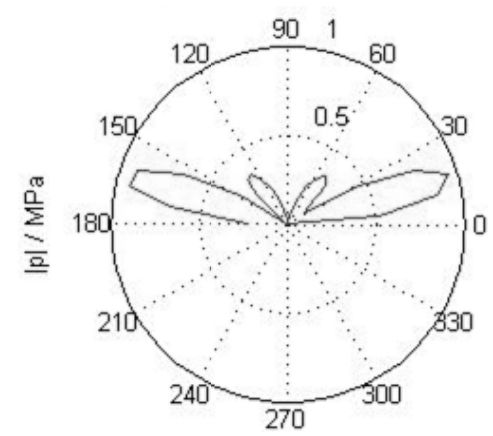

(a) $\mathrm{k}=1$

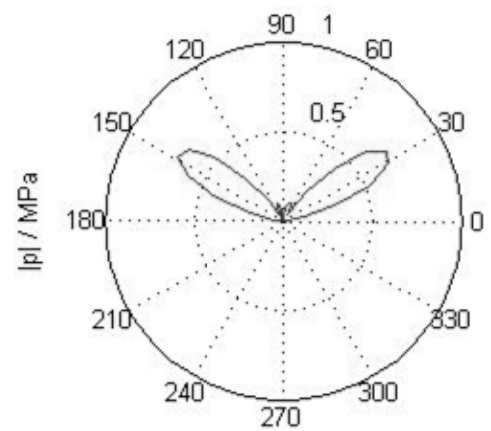

(c) $\mathrm{k}=3$

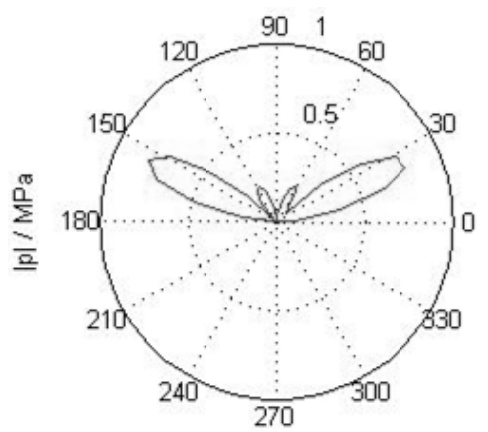

(b) $\mathrm{k}=2$

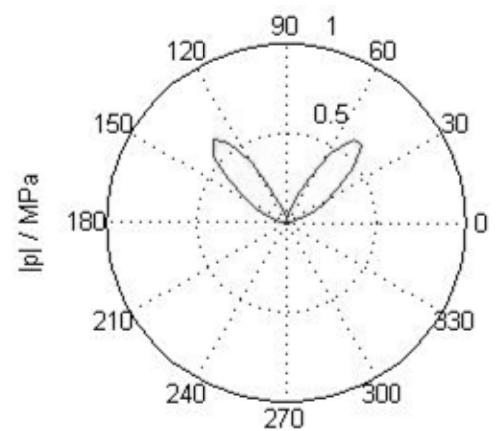

(d) $\mathrm{k}=5$

Figure 5. The directivity of vertical dipole with different harmonic orders at same nature and fundamental frequency 


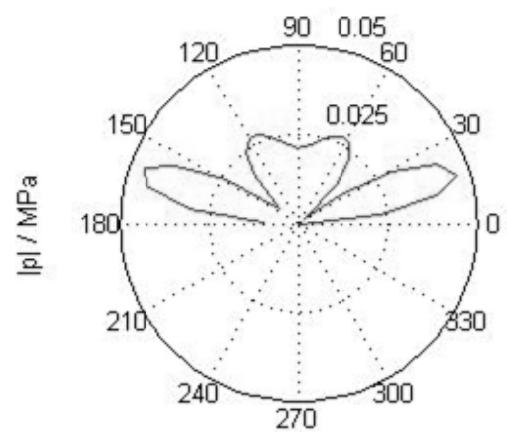

(a) $\mathrm{k}=1$

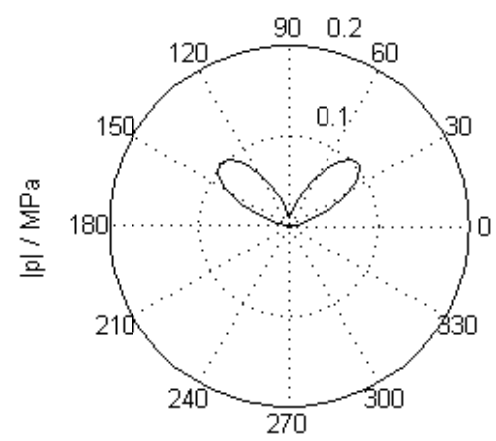

(c) $\mathrm{k}=3$

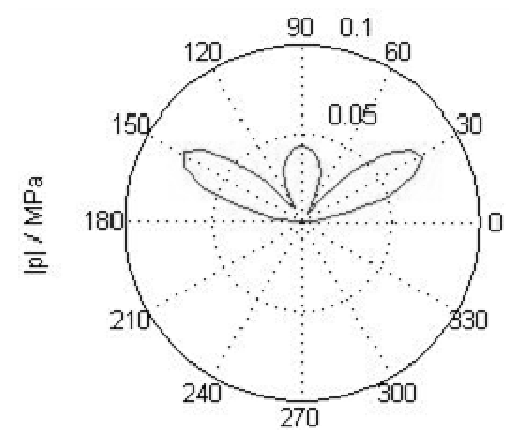

(b) $\mathrm{k}=2$

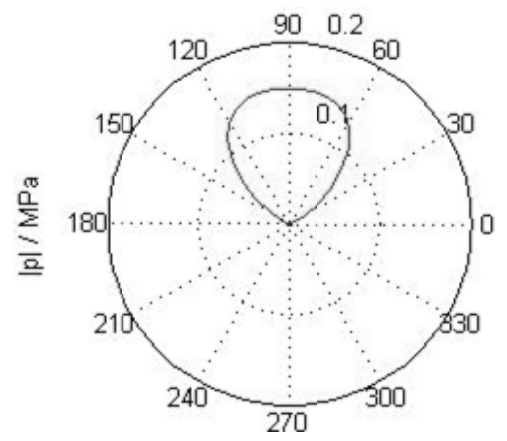

(d) $\mathrm{k}=5$

Figure 6 . The directivity of horizontal dipole with different harmonic orders at same nature and fundamental frequency

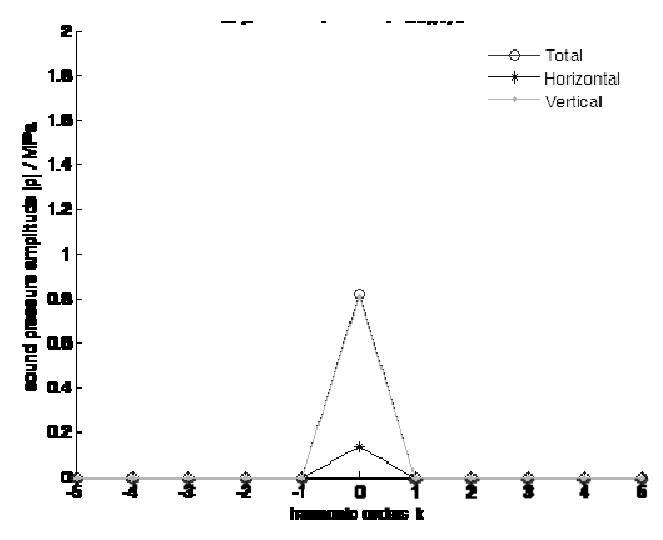

(a) 0

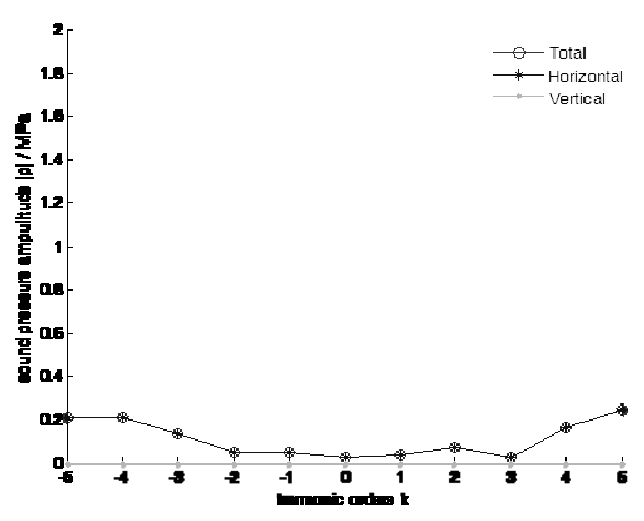

(b) $\pi / 2$

Figure 7. The distribution from horizontal and vertical dipole at different observation angle 